\title{
Is Bilateral Strengthening an Effective Treatment Approach in Patients with Unilateral Lateral Elbow Tendinopathy?
}

\author{
Dimitrios Stasinopoulos $^{1^{*}}$, Antonis Constantinou ${ }^{2}$, Dimitrios Lamnisos ${ }^{3}$ \\ ${ }^{I}$ Dept. of Physiotherapy, Faculty of Health and Caring Sciences, University of West Attica, \\ ${ }^{2}$ Department of Health Sciences, Physiotherapy Program, School of Sciences, European University of Cyprus, \\ Nicosia, Cyprus \\ ${ }^{3}$ School of Sciences, European University of Cyprus, Nicosia, Cyprus
}

*Corresponding Author: Dimitrios Stasinopoulos, Assistant Professor, Physiotherapy, Dept. of Physiotherapy, Faculty of Health and Caring Sciences, University of West Attica, Member of Laboratory of Neuromuscular \& Cardiovascular Study of Motion (LANECASM), Agiou Spyridonos 28, Egaleo 12243, Athens -GREECE

\begin{abstract}
:
Objective: To compare the effectiveness of a heavy-slow resistance exercise programme in patients with unilateral Lateral Elbow Tendinopathy (LET) for the injured limb and a heavy-slow resistance exercise programme in patients with unilateral LET for both limbs.
\end{abstract}

Design: Randomized controlled trial

Research: Research Centre.

Subjects: This study was carried out with 23 patients who had chronic LET. They were randomized into two groups

Interventions: Group A $(n=12)$ was treated with a heavy-slow resistance exercise programme in patients with unilateral LET for the injured limb. A heavy-slow resistance exercise programme in patients with unilateral LET for both limbs was given to group $B(n=12)$. All patients received five treatments per week for six weeks.

Outcomes: Pain was evaluated using a visual analogue scale and function using a visual analogue scale and pain-free grip strength at the end of the six-week course of treatment (week 6) and two months (week 14) after the end of treatment.

Results: There were no significant differences in the magnitude of improvement between the groups at weeks 6 (end of treatment) and 14 (follow up).

Conclusion: there were no significant differences in the magnitude of improvement between the two groups at the end of the treatment and at the follow up.

Keywords: tennis elbow, bilateral strengthening, lateral epicondylitis, lateral elbow tendinopathy, exercise program

\section{INTRODUCTION}

Lateral elbow tendinopathy (LET) seems to be the most appropriate term to use in clinical practice because all the other terms such as lateral epicondylitis, lateral epicondylalgia, lateral epicondylosis and/or tennis elbow make reference to inappropriate aetiological, anatomical and pathophysiological terms ${ }^{1}$. LET is one of the most common lesions of the arm work-related or sport-related pain disorder. The condition is usually defined as a syndrome of pain in the area of the lateral epicondyle ${ }^{2}$ that may be degenerative or failed healing tendon response rather than inflammatory ${ }^{3}$. Hence, the increased presence of fibroblasts, vascular hyperplasia, proteoglycans and glycosaminoglycans together with disorganized and immature collagen may all take place in the absence of inflammatory cells ${ }^{4}$. The most commonly affected structure is the origin of the extensor carpi radialis brevis (ECRB) ${ }^{4}$. The 
dominant arm is commonly affected, the peak prevalence of LET is between 30 and 60 years of age $\mathrm{e}^{2,5}$ and the disorder appears to be of longer duration and severity in women ${ }^{3,6}$.

The main complaints of patients with LET are pain and decreased function ${ }^{2,3}$ both of which may affect daily activities. Diagnosis is simple, and a therapist should be able to reproduce this pain in at least one of three ways: (1) digital palpation on the facet of the lateral epicondyle, (2) resisted wrist extension and/or resisted middle-finger extension with the elbow in extension, and (3) by getting the patient to grip an object ${ }^{2,3,5}$.

Although the signs and symptoms of LET are clear and its diagnosis is easy, to date, no ideal treatment has emerged. Many clinicians advocate a conservative approach as the treatment of choice for LET $^{2,3,7,8}$. Physiotherapy is a conservative treatment that is usually recommended for LET patients $^{2-9}$. A wide array of physiotherapy treatments have been recommended for the management of LET $^{10-14}$. These treatments have different theoretical mechanisms of action, but all have the same aim, to reduce pain and improve function. Such a variety of treatment options suggests that the optimal treatment strategy is not known, and more research is needed to discover the most effective treatment in patients with LET $^{10-14}$.

One of the most common physiotherapy treatments for LET is an exercise programme ${ }^{2-14}$ and more specifically and a heavy-slow resistance exercise programme for the injured limb. Rehabilitation of LET is changing, and now eccentric training is not the only exercise option. Malliaras and his colleagues ${ }^{15}$ concluded that clinicians should consider eccentric-concentric loading alongside or instead of eccentric loading. Martinez-Silvestrini et al ${ }^{16}$ stated that, unlike Achilles tendinopathy, LET is often related to forceful grip activities requiring isometric contraction, which would be more beneficial than the eccentric contraction in LET. Recently, isometric exercises have been recommended to reduce and manage tendon pain, increasing the strength at the angle of contraction without producing inflammatory signs. ${ }^{17}$ The exercise program in LET should include exercises not only for extensor carpi radialis brevis (ECRB) strengthening, the most commonly affected structure, but also for supinator, rotator cuff, and scapular muscle strengthening. ${ }^{18,19}$ Moreover, patients with LET have also reduced proprioception. ${ }^{20}$ Techniques to improve reduced proprioception are also recommended. In addition, tendon neuroplastic training (TNT) is needed to combine isometric or isotonic strength training with an externally paced audio or visual cue. ${ }^{21}$ Finally, stretching has positive effects in the management of tendon injuries such as LET. The heavy-slow resistance exercise programme is individualized on the basis of the patient's description of pain experienced during the procedure ${ }^{22}$.

On the other hand, sensory and motor system deficits are common in the non-injured limb of patients with unilateral LET $^{23}$. This suggests that there could be benefit from rehabilitation that addresses motor and sensory system features on both sides. To our knowledge, there have been no studies to examine the effectiveness of a heavy-slow resistance exercise programme in patients with unilateral LET on both limbs. Therefore, the aim of the present article was to make a comparison of the effects of a heavy-slow resistance exercise programme in patients with unilateral LET for the injured limb and a heavy-slow resistance exercise programme in patients with unilateral LET for both limbs.

\section{METHODS}

A randomized controlled, monocentre trial was conducted in a clinical setting over 36 months to assess the effectiveness of a heavy-slow resistance exercise programme in patients with unilateral LET for the injured limb and a heavy-slow resistance exercise programme in patients with unilateral LET for both limbs. A parallel group design was used because crossover designs are limited in situations where patients are cured by the intervention and do not have the opportunity to receive the other treatments after crossover ${ }^{24}$. Two investigators were involved in the study: (1) a physiotherapist -Ph.D student (AC) who evaluated the patients to confirm the LET diagnosis, performed all baseline and follow-up assessments, and gained informed consent and (2) a physiotherapist, the primary investigator (DS), who administered the treatments. All assessments were conducted by AC who was blind to the patients' therapy group. IS interviewed each patient to ascertain baseline demographic and clinical characteristics, including patient name, sex, age, duration of symptoms, previous treatment, occupation, affected arm and dominant arm. 
Patients over 18 years old who were experiencing lateral elbow pain were examined and evaluated in the Cyprus Musculoskeletal and Sports Trauma Research Centre (CYMUSTREC) located in Nicosia, Cyprus between January 2016 and November 2018. All patients lived in Cyprus, were native speakers of Greek and were either self-referred or referred by their physician or physiotherapist.

Patients were included in the study if, at the time of presentation, they had been evaluated as having clinically diagnosed LET for at least 12 weeks. Patients were included in the trial if they reported (a) pain on the facet of the lateral epicondyle when palpated, (b) less pain during resistance supination with the elbow in 90。 of flexion rather than in full extension and (c) pain in at least two of the following four tests ${ }^{7}$ :

\section{Tomsen test (resisted wrist extension)}

2. Resisted middle finger test

3. Mill's test (full passive flexion of the wrist)

4. Handgrip dynamometer test.

Patients were excluded from the study if they had one or more of the following conditions: (a) dysfunction in the shoulder, neck (radiculopathy) and/or thoracic region; (b) local or generalized arthritis; (c) neurological deficit; (d) radial nerve entrapment; (e) limitations in arm functions; (f) the affected elbow had been operated on and $(\mathrm{g})$ had received any conservative treatment for the management of LET in the 6 weeks before entering the study ${ }^{25-28}$.

All patients received a written explanation of the trial prior to entry into the study. All patients gave signed informed consent to participate in the study. The study was approved by the Topical Research Ethics Committee and access to patients was authorised by the manager of the research centre.

The patients were randomly allocated to two groups by drawing lots. Patients in Group A were treated with heavy-slow resistance exercise programme for the injured limb and patients in Group B were treated with heavy-slow resistance exercise programme for both limbs.

All patients were instructed to use their arm during the course of the study but to avoid activities that irritated the elbow such as grasping, lifting, knitting, handwriting, driving a car and using a screwdriver. They were also told to refrain from taking anti-inflammatory drugs throughout the course of the study. Patient compliance with this request was monitored using a treatment diary.

Communication and interaction (verbal and non-verbal) between the therapist and patient was kept to a minimum, and behaviours sometimes used by therapists to facilitate positive treatment outcomes were purposefully avoided. For example, patients were given no indication of the potentially beneficial effects of the treatments or any feedback on their performance in the pre-application and post-application measurements ${ }^{29}$.

In both groups the elbow was on the bed extended, the forearm pronated, the wrist in extension (and the hand hanging at the edge of the table). From this position, subjects flexed their wrists and then return to the extension (starting position). In the starting position, subjects carried out an isometric contraction of wrist extensors. When the isometric contraction finished the subjects carried out the eccentric - concentric contraction and so on. The exercise involved isolated wrist extension and flexion paced to an external audio/visual cue on the patients' smartphone (PR Metronome; http://eumlab.com/ pro-metronome/). Subjects were to track the movement and listen to the sound of the metronome with their eyes. Each beat was ten seconds apart, so that the pace of the metronome was setted to 6 beats per minute. This allowed a ten second eccentric, concentric and isometric phase $^{30}$.

Both groups performed three sets of 15 repetitions of slow progressive exercises of the wrist extensors at each treatment, with 1-min rest interval between each set. Subjects were informed to continue with the exercise even if they complained of mild pain. However, subjects were informed to stop the exercise if the pain became disabling. The disabling and mild pain were monitored asking the subjects 
to rate the pain on VAS before and after treatment period. The definition of mild pain was below 4 on VAS whereas the definition of disabling pain was above 8 on $\mathrm{VAS}^{22,25,27}$. When subjects were able to carry out the exercise programme without experiencing any discomfort or pain, free weights were used to increase the load.

Both treatment groups were performed static stretching exercises of the wrist extensors. Three times before and three times after the exercises six times totally were repeated the static stretching exercises at each treatment session, with a 30 second rest interval between each repetition. The other hand helped to be performed the static stretching exercises of the wrist extensors. The patient's elbow was placed in extended position, the forearm in pronated position, and the wrist in ulnar deviation and flexion according to the patient's tolerance. 30-45 seconds was holding this position each time and then released $22,25,27,28$.

Furthermore, the scapular and rotator cuff muscles were strengthened. The strengthening exercises were included (i) shoulder lateral rotation and medial with the elbow in $90^{\circ}$ and $0^{\circ}$ of abduction; (ii) shoulder abduction to $90^{\circ}$ with flexed elbow: (iii) scaption and (iv) diagonal pattern from flexion to extension $^{31}$. Upper trapezius, rhomboids, serratus anterior and levator scapulae were also strengthened ${ }^{32}$. Each exercise were carried out twice at each treatment with 12 repetitions in each set and $1 \mathrm{~min}$ rest interval between each $\operatorname{set}^{33}$. Subjects were informed to continue with the exercise even if they complained of mild pain. However, subjects were informed to stop the exercise if the pain became disabling. When subjects were able to carry out the strengthening exercises without experiencing any discomfort or pain, the load were increased using therabands or free weights.

Finally, the supinator was strengthened. Strengthening exercises of the supinator were carried out with the elbow extended on the table, the forearm pronated, the wrist in mid - position and the hand hanging over the edge of the table. From this position, the patients supinated their arm slowly while counting to 15 using chronometer, then return to the starting position (pronation) ${ }^{34}$.

The above reported exercise programme will be followed five times per week for 6 weeks and will be individualized on the basis of the patient's description of pain experienced during the process.

In group B patients followed the exercise program for both limbs, injured and non-injured.

Pain, function and drop-out rate were measured in the present study. Each patient was evaluated at the baseline (week 0), at the end of treatment (week 6) and at 2 months (week 14) after the end of treatment.

Pain was measured on a visual analogue scale (VAS), where $0(\mathrm{~cm})$ was "least pain imaginable" and $10(\mathrm{~cm})$ was "worst pain imaginable". The pain VAS was used to measure the patient's worst level of pain over the previous $24 \mathrm{~h}$ before each evaluation, and this approach has been shown to be valid and sensitive of the $\mathrm{VAS}^{35}$.

Function was measured using a VAS, in which $0(\mathrm{~cm})$ was taken as "no function" and $10(\mathrm{~cm})$ as "full function". Patients were instructed to report their overall level of elbow function over the previous 24 $\mathrm{h}$ before each evaluation, and this approach has been shown to be valid and sensitive of the VAS ${ }^{35}$.

In addition, function was measured by pain-free grip strength. Pain-free grip strength is defined as the amount of force each patient is able to generate with an isometric gripping action before eliciting pain $^{29}$. Force was measured in pounds with a Jamar hand dynamometer (Figure 1) that had adjustable handles to accommodate different hand sizes. The arm was placed in a standardised position of elbow extension, forearm pronation and internal rotation of the upper limb such that the palmar aspect of the hand faced posteriorly with the upper limb placed by the patient's side. Patients were then instructed to squeeze the dynamometer handles until they first experienced pain and then to release their grip ${ }^{29}$. The attained grip force was subsequently recorded, and the reading was not visible to the patient. Three measures of pain-free grip strength were recorded with a 30 seconds rest interval between each measurement, and the mean value of these repetitions was calculated. 


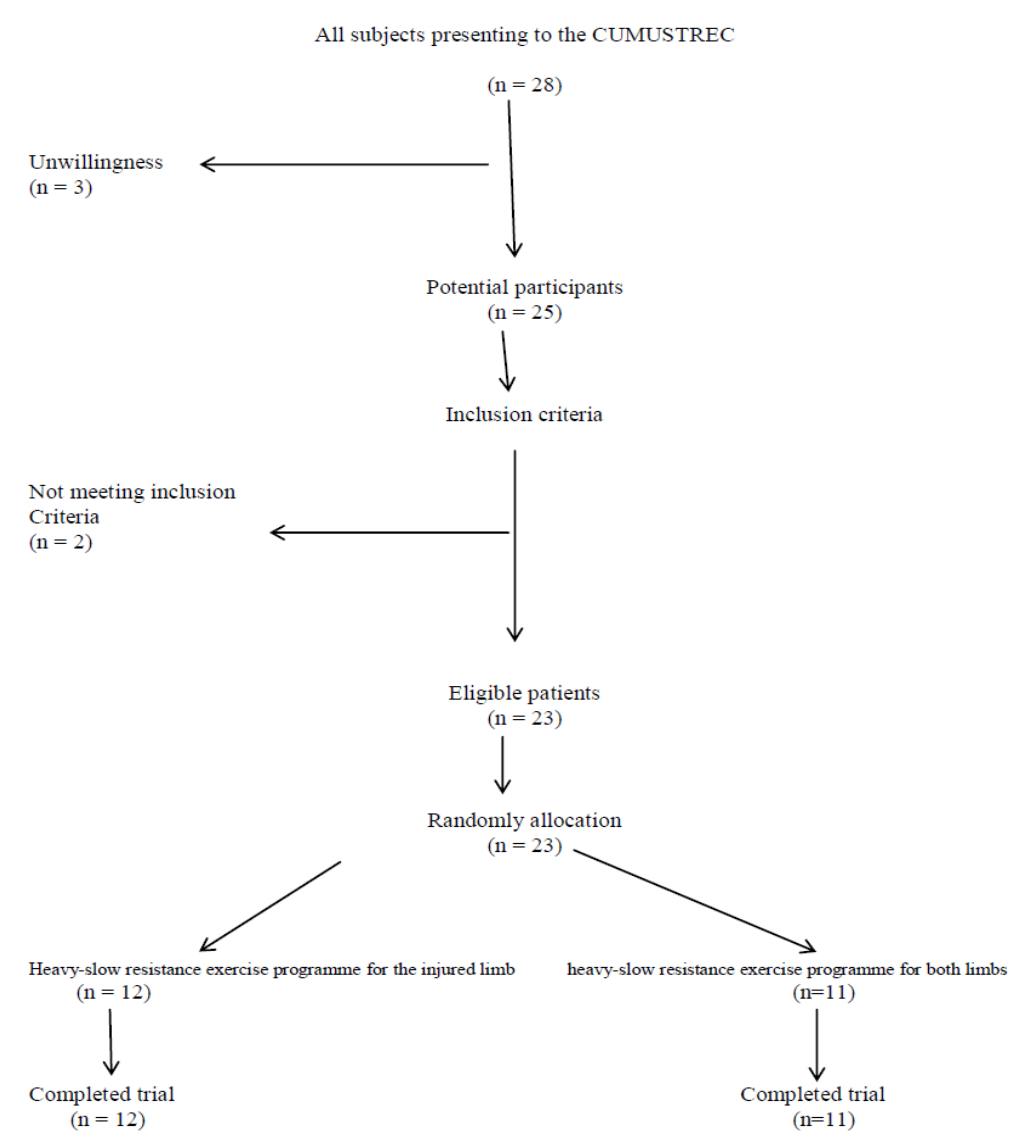

Figure1. Flow chart of the study.

A drop-out rate was also used as an indicator of treatment outcome. Reasons for patient drop out were categorised as follows: (1) a withdraw without reason; (2) not returned for follow-up and (3) request for an alternative treatment.

The change from baseline was calculated for each follow-up. Differences between groups were determined using the independent $t$ test. The difference within groups between baseline and end of treatment was analysed with a paired t test. A 5\% level of probability was adopted as the level for statistical significance. SPSS V.21.00 statistical software was used for the statistical analysis.

\section{RESULTS}

Twenty-eight patients eligible for inclusion visited the centre within the trial period. Three were unwilling to participate in the study, and 2 did not meet the inclusion criteria described above. The other 23 patients were randomly allocated to one of the two possible groups: (a) heavy-slow resistance exercise programme for the injured $\operatorname{limb}(\mathrm{n}=12$; 4 men, 8 women; mean (SD) age 45.56 (5.42) years); (b) heavy-slow resistance exercise programme for both limbs ( $\mathrm{n}=11 ; 3$ men, 8 women; mean (SD) age 44.96 (5.18) years). Patient flow through the trial is summarised in a CONSORT flow chart (fig 1).

At baseline, there were more women in the groups (nine more in total). The mean age of the patients was about 45 years, and the duration of LET was about 6 months. LET was in the dominant arm in all patients $(100 \%)$. There were no significant differences in mean age ( $p>0.0005$, independent $t$ test) or the mean duration of symptoms ( $>0.0005$, independent $t$ test) between the groups. Patients had received a wide range of previous treatments (table 1). Drug therapy had been tried by more than $70 \%$. All patients were manual workers.

Table1. Previous treatments of participants

\begin{tabular}{|l|l|l|}
\hline & $\begin{array}{l}\text { Heavy-slow resistance exercise programme for the } \\
\text { injured limb }(\%)\end{array}$ & $\begin{array}{l}\text { Heavy-slow resistance exercise } \\
\text { programme for both limbs (\%) }\end{array}$ \\
\hline Drugs & $9(76 \%)$ & $8(72 \%)$ \\
\hline Physiotherapy & $2(16 \%)$ & $3(28 \%)$ \\
\hline Injection & $1(8 \%)$ & \\
\hline
\end{tabular}

Values are number (\%). 
Is Bilateral Strengthening an Effective Treatment Approach in Patients with Unilateral Lateral Elbow Tendinopathy?

Baseline pain on VAS was 8.80 (95\% CI 8.45 to 8.98 ) for the whole sample ( $\mathrm{n}=23$; table 2$)$. There were no significant differences between the groups for baseline pain ( $>0.05$ independent $t$ test; table 2). At week 6, there was a decline in VAS of about 8 units in the heavy-slow resistance exercise programme for both limbs and 7.50 units in the heavy-slow resistance exercise programme for the injured limb compared with the baseline $(\mathrm{p}<0.0005$, paired t test; table 3$)$. There were no significant differences in the magnitude of reduction between the groups at weeks 6 and 14 ( $<<0.0005$ independent $\mathrm{t}$ test; table 3 ).

Baseline function on VAS was 3.70 (95\% CI 3.31 to 4.52) for the whole sample ( $\mathrm{n}=23$; table 2). There were no significant differences between the groups for baseline function $(p>0.05$ independent $t$ test; table 2). At week 6, there was a rise in VAS of approximately 4.70 units in heavy-slow resistance exercise programme for both limbs and 4.10 units in the heavy-slow resistance exercise programme for the injured limb compared with the baseline $(\mathrm{p}<0.0005$, paired $t$ test; table 3$)$. There were no significant differences in the magnitude of improvement between the groups at weeks 6 and 14 $(\mathrm{p}<0.0005$ independent $\mathrm{t}$ test; table 3$)$.

Baseline pain-free grip strength was $24.8 \mathrm{lb}$ (95\% CI 23.95 to 26.43) for the whole sample ( $\mathrm{n}=23$; table 2). There were no significant differences between the groups for baseline pain-free grip strength ( $>0.05$ independent $t$ test; table 2). At week 6 , there was a rise in pain-free grip strength of approximately 37 units in the heavy-slow resistance exercise programme group and 31 units in the heavy-slow resistance exercise programme for the injured limb group compared with the baseline $(\mathrm{p}<0.0005$, paired $\mathrm{t}$ test; table 3$)$. There were no significant differences in the magnitude of improvement between the groups at weeks 6 and 14 ( $\mathrm{p}<0.0005$ independent $\mathrm{t}$ test; table 3$)$.

There were no drop-outs and all patients successfully completed the study.

Table2. Pain, function and pain-free grip strength over the 24 before each evaluation

\begin{tabular}{|c|c|c|c|c|c|c|}
\hline & \multicolumn{2}{|l|}{ PAIN $(\mathrm{cm})$} & \multicolumn{2}{|l|}{ Function $(\mathrm{cm})$} & \multicolumn{2}{|c|}{ Pain Free grip strength (lb) } \\
\hline & Injured limb & Both limbs & Injured limb & Both limbs & Injured limb & Both limbs \\
\hline Week 0 & $\begin{array}{l}8.85 \text { (8.40 to } \\
8.97)\end{array}$ & $\begin{array}{l}8.75 \text { (8.28 to } \\
8.83)\end{array}$ & $\begin{array}{l}3.65 \text { (3.26 to } \\
4.11)\end{array}$ & $\begin{array}{l}3.75 \text { (3.44 to } \\
4.25)\end{array}$ & $\begin{array}{l}24.75(24.32 \\
\text { to } 25.46)\end{array}$ & $\begin{array}{l}24.85(24.52 \\
\text { to } 25.32)\end{array}$ \\
\hline Week 6 & $\begin{array}{l}1.35 \text { (1.12 to } \\
1.86)\end{array}$ & $\begin{array}{l}0.75(0.54 \text { to } \\
1.29)\end{array}$ & $\begin{array}{l}7.75 \text { (7.44 to } \\
8.17)\end{array}$ & $\begin{array}{l}8.45 \text { (7.97 to } \\
8.82)\end{array}$ & $\begin{array}{l}55.75(55.46 \\
\text { to } 56.02)\end{array}$ & $\begin{array}{l}61.85(61.49 \\
\text { to } 62.18)\end{array}$ \\
\hline Week 14 & $\begin{array}{l}1.32(1.09 \text { to } \\
1.82)\end{array}$ & $\begin{array}{l}0.71(0.50 \text { to } \\
1.35)\end{array}$ & $\begin{array}{l}7.92(7.53 \text { to } \\
8.31)\end{array}$ & $\begin{array}{l}8.51(8.02 \text { to } \\
8.91)\end{array}$ & $\begin{array}{l}56.18(55.84 \\
\text { to } 56.77)\end{array}$ & $\begin{array}{l}62.25(61.93 \\
\text { to } 62.64)\end{array}$ \\
\hline
\end{tabular}

Table3. Change in pain, function, and pain-free grip strength over the $24 h$ before each evaluation from baseline.

\begin{tabular}{|l|l|l|l|l|l|l|l|}
\hline & \multicolumn{2}{|l|}{ PAIN $(\mathrm{cm})$} & \multicolumn{2}{l|}{ Function $(\mathrm{cm})$} & Pain Free grip strength (lb) & \\
\hline & Injured limb & Both limbs & Injured limb & Both limbs & Injured limb & Both limbs & p Value \\
\hline Week 6 & -7.50 & -8.00 & +4.10 & +4.70 & +31 & +37 & $<0.0005$ \\
\hline $\begin{array}{l}\text { Week } \\
14\end{array}$ & -7.53 & -8.04 & +4.27 & +4.76 & +31.43 & +37.40 & $<0.0005$ \\
\hline
\end{tabular}

\section{DISCUSSION}

The results obtained from this randomized clinical trial are novel, as to date there have been no data comparing the effectiveness of a heavy-slow resistance exercise programme in patients with unilateral LET for the injured limb and a heavy-slow resistance exercise programme in patients with unilateral LET for both limbs. Although the heavy-slow resistance exercise programme in patients with unilateral LET for both limbs produced the largest effect at the end of treatment and at the follow up, there were no significant differences in the magnitude of improvement between the groups at weeks 6 (end of treatment) and 14 (follow up).

Alfredson et al. (1998) ${ }^{36}$ were first proposed the eccentric training of the injured tendon. It is the most commonly used conservative approach in the treatment of tendinopathy. Malliaras and his colleagues $(2013)^{15}$ concluded that clinicians should consider eccentric-concentric loading alongside or instead of eccentric loading in Achilles and patellar tendinopathy. A Heavy Slow Resistance (HSR) program is recommended in the management of lower limb tendinopathy ${ }^{37,38}$. The HSR program was produced equivalent pain and function improvement (VISA) than the Alfredson eccentric program, but 
significantly better patient satisfaction at six month follow -up. In the Achilles tendon, eccentric and HSR have recently been shown to yield similar clinical outcomes (VISA and patient satisfaction) at 1 year follow up. Based on the above findings, the HSR program can be recommended as an alternative to the Alfredson eccentric program lower limb tendinopathy rehabilitation. Studies determining the effectiveness of such as exercises at other tendinopathies such as LET are needed.

Systematic review ${ }^{39}$ and $\mathrm{RCT}^{40}$ favor eccentric over other types of contractions in the management of LET, but Martinez-Silvestrini et al. (2005) ${ }^{16}$ stated that, unlike Achilles tendinopathy, LET is often related to forceful grip activities requiring isometric contraction, which would be more beneficial than eccentric contraction in LET. Recently, isometric exercises have been recommended to reduce and manage tendon pain increasing the strength at the angle of contraction without producing inflammatory signs ${ }^{41}$. Five repetitions of 45 -second isometric mid-range quadriceps exercise at $70 \%$ of maximal voluntary contraction have been shown to reduce patellar tendon pain for 45 minutes post exercise and this was also associated with a reduction in motor cortex inhibition of the quadriceps that was associated with patellar tendinopathy ${ }^{21}$. The dosage of isometric contractions in the present was based on clinical experience $e^{41-43}$ and their effect on pain in patients with LET requires further study. Therefore, it was hypothesized that the simultaneous use of these two kinds of contractions (isotonic and isometric) and static stretching exercises will further enhance the analgesic effect of contractions in the treatment of LET, increasing the arm function.

In LET not only the ECRB (injured tendon) but also the supinator may be involved ${ }^{1}$. The exercise program should include exercises not only for ECRB strengthening but also for supinator strengthening ${ }^{18}$. In addition, rotator cuff and scapular muscles strengthening is also needed ${ }^{19}$. Based mainly on clinical experience, supinator, rotator cuff and scapular muscles weakness in LET patients is commonly addressed as increasing pain, and decreasing functional ability and hand - grip strength. This means that the causes of LET may not be limited to the ECRB. Functional impingement of the supinator, rotator cuff and scapular muscles due to altered joint mechanism and muscle imbalance can impair the stabilization of the elbow resulting in overcompensation of the ECRB. This may lead to micro trauma of the soft tissue structures present at the lateral epicondyle thus causing symptoms of LET $^{18,19}$. It is reasonable that enhancements with gripping might have happened from a blend of enhanced motor control and upgraded muscular power of the supinator, rotator cuff and scapular muscles. Changes in the supinator, rotator cuff and scapular muscles may lead to altered and compensatory changes in the ECRB that may overload the ECRB during repetitive movements, thus causing symptoms of LET $^{18,19}$. Using supinator, rotator cuff and scapular muscles strengthening loading, usual motion might have been returned, resulting in resolution of pain with actions and a return to painless gripping for the patient.

Therapists use techniques to improve symptoms (pain and function) while LET patients have also reduced proprioception ${ }^{20}$. Unfortunately, therapists ignore the reduced proprioception in the management of LET. If therapists use modalities to improve the proprioception, the results will be effective sooner. However, more research is needed to find out the factors for the development and progression of proprioception in LET patients. In addition, future studies are needed to investigate which treatment is the most effective to increase LET proprioception and if this treatment is effective for all LET patients' for example LET patients with neck stiffness or patients with acute LET will have benefit from proprioception training. More research is also needed to find out which joint, wrist, elbow or shoulder, has poor proprioception in LET patients and how this reduced proprioception affects the management of LET. Finally, further trials are needed to clarify whether reduced proprioception plays a role in the development of LET, the progression of the degenerative changes found in LET, and in the large recurrence rate of LET.

Recent evidence suggests that the central nervous system may play a role in the management of tendinopathy ${ }^{44}$. TNT is proposed to address the central nervous system component of tendinopathy ${ }^{21}$. Compared to other conservative therapies, TNT addresses the motor control deficits present in tendinopathy ${ }^{21}$.

Tendon stretching is an important factor in the rehabilitation of LET patients and must be included in the rehabilitation program. Stretching may strengthen the tendon or make it more resistant to strain and increase the range of motion of the relevant joint ${ }^{45,46}$. In addition, stretching may also be 
contributed with a "lengthening" of the muscle-tendon unit, orientation of the new collagen fibres and subsequently less stress exerted during joint movement ${ }^{36}$.

Sensory and motor system deficits are common in the non-injured limb of patients with unilateral $\mathrm{LET}^{23}$. This suggests that there could be benefit from rehabilitation that addresses motor and sensory system features on both sides. Specific training of the contralateral limb may also provide additional benefits to the affected limb through cross education ${ }^{23}$. Cross education is a process that unilateral exercise leads to strength and skill adaptations bilaterally ${ }^{47}$. Although the heavy-slow resistance exercise programme in patients with unilateral LET for both limbs produced the largest effect at the end of treatment and at the follow up in the present study, there were no significant differences in the magnitude of improvement comparing with the heavy-slow resistance exercise programme in patients with unilateral LET for the injured limb.

The load of exercises was increased according to the patients' symptoms otherwise the results are poor $^{48}$. Furthermore, eccentric exercises were performed at a low speed in every treatment session because this allows tissue healing ${ }^{4}$. Ice was recommended at the end of the treatment but research has shown that ice as a supplement to an eccentric exercise programme offers no benefit to patients with tendinopathy ${ }^{25}$. Finally, the avoidance of painful activities is crucial for tendon healing, because training during the treatment period increases patients' symptoms and delays tendon healing ${ }^{49}$.

Eccentric exercises appears to reduce the pain and improve function, reversing the pathology of LET $^{48,50,51}$ as supported by experimental studies on animals ${ }^{52}$. The way that eccentric training achieves the goals remains uncertain, as there is a lack of good quality evidence to confirm that physiological effects translate into clinically meaningful outcomes and vice versa. The clinical improvement of the HSR group was accompanied by increased collagen turnover. It is unknown if the isometric contractions can reverse the pathology of the tendinopathy and in this case the pathology of LET or reduce only the pain.

However, this trial does have some shortcomings. First, the sample was small. Second, no placebo (sham) or no treatment group was included in the present trial. The placebo (sham)/no treatment group is important when the absolute effectiveness of a treatment is determined. However, the absolute effectiveness of technique based interventions is difficult to investigate because a good and trustworthy placebo (sham)/no treatment control for exercise programmes appears to be difficult or impossible to devise, due in part to difficulties in defining the active element of these treatments. Absolute effectiveness also does not provide the therapists with information as to which is the most appropriate treatment for the management of a condition, in this case LET. Third, other activities treatments patients might be getting when not in the centr were not monitored. Patients' diaries suggested that patients were compliant to the study instructions, although patients may have given incorrect details to please the investigators. For example, it was possible that patients followed the treatment but took analgesic medications at the same time, and the improvement of symptoms may be due to those medications. Therefore, ways should be found to measure how other treatments such as analgesic medications contribute to the improvement of symptoms. Finally, the blinding of patients and therapists would be problematic in that case, if not impossible, because patients know if they are receiving the exercise programme treatment and therapists need to be aware of the treatment to administer it appropriately. In addition to the previous reported weaknesses, structural changes in the tendons related to the treatment intervention were not shown, and the intermediate and long-term effects (6 months or more after the end of treatment) of treatments were not investigated. Further research is needed to establish the possible mechanism of action of this treatment approach, and the cost-effectiveness of such treatment, because reduced cost is an important issue for the recommendation of any given treatment.

In conclusion, a heavy-slow resistance exercise programme in patients with unilateral LET for the injured limb and a heavy-slow resistance exercise programme in patients with unilateral LET for both limbs reduced pain and improved function at the end of the treatment and at the follow-up. The heavy-slow resistance exercise programme in patients with unilateral LET for both limbs produced the largest effect at the end of the treatment and at follow-up. However, there were no significant differences in the magnitude of improvement comparing to the heavy-slow resistance exercise programme in patients with unilateral LET for the injured limb. Future well designed studies are needed to confirm the results of the present trial. 
Is Bilateral Strengthening an Effective Treatment Approach in Patients with Unilateral Lateral Elbow Tendinopathy?

\section{FUNDING}

This research received no specific grant from any funding agency in the public, commercial, or not for- profit sectors.

\section{REFERENCES}

[1] Stasinopoulos D, Johnson MI. Lateral elbow tendinopathy is the most appropriate diagnostic term for the condition commonly referred to as lateral epicondylitis. Medical Hypotheses 2006; 67: 1399-1401.

[2] Bisset LM, Vicenzino B. Physiotherapy management of lateral epicondylalgia. J Physiother 2015; 61: 174-81

[3] Coombes BK, Bisset L, Vicenzino B. Management of Lateral Elbow Tendinopathy-One Size Does Not Fit All. J Orthop Sports Phys Ther 2015; 17:1-38.

[4] Kraushaar B, Nirschl R. Current concepts review - tendinosis of the elbow (tennis elbow). Clinical features and findings of histological immunohistochemical and electron microscopy studies. J Bone Joint Surg Am 1999; 81: 259 - 85.

[5] Vicenzino B, Wright A. Lateral epicondylalgia I: epidemiology, pathophysiology, aetiology and natural history. Phys Ther Rev 1996; 1: 23 - 34.

[6] Waugh E, Jaglal S, Davis A, Tomlinson G, Verrier M. Factors associated with prognosis of lateral epicondylitis after 8 weeks of physical therapy. Arch Phys Med Rehabil 2004; 85: 308 - 18.

[7] Haker E. Lateral epicondylalgia: diagnosis, treatment and evaluation. Crit Rev Phys Rehabil Med 1993; 5: $129-54$.

[8] Hong QN, Durand MJ, Loisel P. Treatment of lateral epicondylitis where is the evidence? Joint Bone Spine2004; 71: 369-373.

[9] Stasinopoulos D, Johnson MI. Physiotherapy and tennis elbow/lateral epicondylitis. Letter. Rapid response to Assendelft et al. (2003) article Tennis elbow: BMJ, 6 September 2004.

[10] Trudel D, Duley J, Zastrow I, Kerr E, Davidson R, MacDermid J. Rehabilitations for patients with lateral epicondylitis: a systematic review. J Hand Ther 2004; 17: 243 - 66.

[11] Smidt N, Assendelft W, Arola $\mathrm{H}$ et al. Effectiveness of physiotherapy for lateral epicondylitis: a systematic review. Ann Med 2003; 35: 51 - 62.

[12] Labelle H, Guibert R, Joncas J, Newman N, Fallaha M, Rivard C. Lack of scientific evidence for the treatment of lateral epicondylitis of the elbow: an attempted meta-analysis. J Bone Joint Surg 1992; 74: $646-51$.

[13] Wright A, Vicenzino B. Lateral epicondylalgia II: therapeutic management. Phys Ther Rev 1997; 2: 39 - 48.

[14] Bisset L, Paungmali A, Vicenzino B, et al. A systematic review and meta-analysis of clinical trials on physical interventions for lateral epicondylalgia. British Journal of Sports Medicine 2005; 39: 411-422.

[15] Malliaras P, Barton C, Reeves N, Langberg H. Achilles and patellar tendinopathy loading programmes. A systematic review comparing clinical outcomes and identifying potential mechanisms for effectiveness. Sports Med 2013; 43:267-86.

[16] Martinez-Silvestrini JA, Newcomer KL, Gay RE, Schaefer MP, Kortebein P, Arendt KW. Chronic lateral epicondylitis: comparative effectiveness of a home exercise program including stretching alone versus stretching supplemented with eccentric or concentric strengthening. J Hand Ther 2005; 18:411-9.

[17] Malliaras P, Cook J, Purdam C, et al. Patellar tendinopathy: clinical diagnosis, load management, and advice for challenging case presentations. J Orthop Sports Phys Ther 2015; 45:887-898.

[18] Stasinopoulos D. Strengthening of supinator in the management of Lateral Elbow Tendinopathy. AMJ 2017; 10:373-374.

[19] Stasinopoulos D. Scapular and rotator cuff strengthening in patients with lateral elbow tendinopathy. Hong Kong Physiother J 2017; 37:25-26.

[20] Juul-Kristensen B, Lund H, Hansen K, et al. Poorer elbow proprioception in patients with lateral epicondylitis than in healthy controls: a cross-sectional study. J Shoulder Elbow Surg 2008;17:72S-81S

[21] Rio E, Kidgell D, Moseley GL, et al. Tendon neuroplastic training: changing the way we think about tendon rehabilitation: a narrative review. Br J Sports Med 2015; 50:209-215.

[22] Stasinopoulos D, Stasinopoulos I. Comparison of effects of eccentric training, eccentric-concentric training, and eccentric-concentric training combined with isometric contraction in the treatment of lateral elbow tendinopathy. J Hand Ther 2017; 30:13-19.

[23] Heales LJ, Lim EC, Hodges PW, Vicenzino B. Sensory and motor deficits exist on the non-injured side of patients with unilateral tendon pain and disability--implications for central nervous system involvement: a systematic review with meta-analysis. Br J Sports Med 2014; 48(19):1400-6.

International Journal of Sports and Physical Education (IJSPE) 
Is Bilateral Strengthening an Effective Treatment Approach in Patients with Unilateral Lateral Elbow Tendinopathy?

[24] Johannsen F, Gam A, Hauschild B, Mathiesen B, Jensen L. Rebox: an adjunct in physical medicine? Arch Phys Med Rehabil 1993; 74: 438 - 40.

[25] Manias P, Stasinopoulos D. A controlled clinical pilot trial to study the effectiveness of ice as a supplement to the exercise programme for the management of lateral elbow tendinopathy. British Journal of Sports Medicine 2006; 40: 81-85.

[26] Pienimaki T, Tarvainen T, Siira P, Vanharanta H. Progressive strengthening and stretching exercises and ultrasound for chronic lateral epicondylitis. Physiotherapy 1996; 82: 522_- 30.

[27] Stasinopoulos D, Stasinopoulos I. Comparison of effects of Cyriax physiotherapy, a supervised exercise programme and polarized polychromatic non-coherent light (Bioptron light) for the treatment of lateral epicondylitis. Clinical Rehabilitation 2006; 20: 12-23.

[28] Stasinopoulos D, Stasinopoulos I, Manias P, Stasinopoulou K. Comparison of effects of a home exercise programme and a supervised exercise programme for the management of lateral elbow tendinopathy, British Journal of Sports Medicine 2010; 44: 579-583.

[29] Vicenzino B, Wright A, Collins C. The initials effects of a cervical spine manipulative physiotherapy treatment on the pain and dysfunction of lateral epicondylalgia. Pain 1996; 68: 69-74.

[30] Welsh P. Tendon neuroplastic training for lateral elbow tendinopathy: 2 case reports. J Can Chiropr Assoc 2018; 62(2): 98-104

[31] Bhatt JB, Glaser R, Chavez A, Yung E. Middle and lower trapezius strengthening for the management of lateral epicondylalgia: a case report. J Orthop Sports Phys Ther 2013;43(11):841-7.15.

[32] Sharma M, Eapen C, Kamath J. Effect of adding rotator cuff strengthening to therapeutic ultrasound and wrist extensor eccentric exercise for lateral epicondylalgia - a randomized clinical trial. Int J Health Sci Res. 2015; 5(7):250-257.

[33] Stasinopoulos D, Stasinopoulos I, Stasinopoulou K. A pilot trial to study the effectiveness of an exercise programme in the treatment of rotator cuff tendinopathy. Biology of exercise 2014; 10: 69-77.

[34] Demosthenous M., Stasinopoulos D, Lamnisos D. Comparison the effectiveness of eccentric - concentric training of wrist extensors and eccentric - concentric training combined with supinator strengthening in healthy population J Orthop Res Physiother 2017;3: 036.

[35] Stratford P, Levy D, Gauldie S, Levy K, Miseferi D. Extensor carpi radialis tendonitis: a validation of selected outcome measures. Physiother Can 1987; 39: 250 - 55.

[36] Alfredson H, Pietila T, Johnson P, Lorentzon R. Heavy load eccentric calf muscle training for the treatment of chronic Achilles tendinosis. Am J Sports Med 1998; 26: 360 - 366.

[37] Beyer R, Kongsgaard M, Hougs Kjær B, Øhlenschlæger T, Kjær M, Magnusson SP. Heavy Slow Resistance versus Eccentric Training as Treatment for Achilles Tendinopathy: A Randomized Controlled Trial_.Am J Sports Med 2015; 43:1704-11.

[38] Kongsgaard M, Kovanen V, Aagaard P, Doessing S, Hansen P, Laursen AH, Kaldau NC, Kjaer M, Magnusson SP. Corticosteroid injections, eccentric decline squat training and heavy slow resistance training in patellar tendinopathy. Scandinavian Journal of Medicine \& Science in Sports 2009; 19:790802.

[39] Raman J, MacDermid JC, Grewal R. Effectiveness of different methods of resistance exercises in lateral epicondylosis-a systematic review. $J$ Hand Ther 2012; 25:5-25.

[40] Peterson M, Butler S, Eriksson M, Svardsudd K. A randomized controlled trial of eccentric vs. concentric graded exercise in chronic tennis elbow (lateral elbow tendinopathy). Clin Rehabil 2014; 28:862-72.

[41] Malliaras P, Cook J, Purdam C, Rio E. Patellar Tendinopathy: Clinical Diagnosis, Load Management, and Advice for Challenging Case Presentations. J Orthop Sports Phys Ther 2015; Sep 21:1-33.

[42] Rio E, Kidgell D, Purdam C, Gaida J, Moseley GL, Pearce AJ, Cook J. Isometric exercise induces analgesia and reduces inhibition in patellar tendinopathy. Br J Sports Med 2015; 49:1277-83.

[43] Stasinopoulos D. The Effectiveness of Isometric Contractions Combined with Eccentric Contractions and Stretching Exercises on Pain and Disability in Lateral Elbow Tendinopathy. A case Report. Journal of Novel Physiotherapies 2015; 5:1-5.

[44] Plinsinga ML, Brink MS, Vicenzino B, van Wilgen P. Evidence of nervous system sensitization in commonly presenting and persistent painful tendinopathies: a systematic review. J Orthop Sport Phys Ther 2015; 45(11):864-876.

[45] Silva RS, Nakagawa TH, Ferreira AL, et al. Lower limb strength and flexibility in athletes with and without patellar tendinopathy. Physical Therapy in Sport 2016; 31:20:19-25.

[46] Stanish WD, Curwin S, Mandell S. Tendinitis: its etiology and treatment. Oxford University Press; 2000 Oct 12 . 
Is Bilateral Strengthening an Effective Treatment Approach in Patients with Unilateral Lateral Elbow Tendinopathy?

[47] Lee M, Carroll TJ. Cross education: possible mechanisms for the contra lateral effects of unilateral resistance training. Sports Med 2007; 37:1-14.

[48] Jensen K, Di Fabio R. Evaluation of eccentric exercise in treatment of patellar tendinitis. Physical Therapy 1989; 69: 211-216.

[49] Visnes, H, Cook J, Bahr R. No effect of eccentric training on Jumper's knee in volleyball players during the competitive season. A randomized clinical trial. Clin J Sports Med 2005; 15: 227-34.

[50] Khan KM, Cook JL, Kannus P, et al. Time to abandon the "tendonitis" myth. BMJ 2002; 324:626-7.

[51] Hawary R, Stanish W, Curwin S. Rehabilitation of tendon injuries in sport. Sports Med 1997; 24:347-58.

[52] Khan K, Cook J, Taunton J, et al. Overuse tendinosis, not tendinitis: a new paradigm for a difficult clinical problem. Phys Sportsmed 2000; 28:38-48.

Citation: Dimitrios Stasinopoulos, et.al. "Is Bilateral Strengthening an Effective Treatment Approach in Patients with Unilateral Lateral Elbow Tendinopathy?" International Journal of Sports and Physical Education (IJSPE), vol 6, no. 2, 2020, pp. 9-19. doi: http://dx.doi.org/10.20431/2454-6380.0602002.

Copyright: (C) 2020 Authors. This is an open-access article distributed under the terms of the Creative Commons Attribution License, which permits unrestricted use, distribution, and reproduction in any medium, provided the original author and source are credited. 